\title{
Schwerpunkt: Ganztagsschule
}

\section{Uwe Sander/Heinz-Hermann Krüger/Thomas Rauschenbach}

\section{Editorial}

Die Debatte um Ganztagsschulen steht in der Bundesrepublik Deutschland unter einem hohen Erwartungsdruck. So soll die Erweiterung der täglichen Schulzeit über den frühen Nachmittag hinaus sowohl den Unterricht effektiver und schülerfreundlicher gestalten als auch die individuelle Förderung ermöglichen sowie die Kreativität von Schule erhöhen. Diese Hoffnungen werden zusammengefasst in der Zielsetzung einer neuen schulischen Lehr- und Lernkultur, die auf Bundesebene ausdrücklich mit dem Label „Bildungsreform" assoziiert wird. Neben schulbezogenen Intentionen verfolgt das Projekt der Ganztagsschule in Hinblick auf die Eltern eine bessere Vereinbarung von Kindern und Beruf; gleichwohl soll die Ganztagsschule aber auch mehr Elternengagement in der und für die Schule ihrer Kinder erzeugen.

Wie sich nun tatsächlich, jenseits von Sollensbekundungen, die bundesdeutsche Situation der Ganztagsschule darstellt, und zwar auch in international vergleichender Perspektive, dazu geben die Beiträge dieser Ausgabe der ,Zeitschrift für Erziehungswissenschaft" Auskunft.

Heinz Günter HOLTAPPELS beschreibt in seinem Stichwortartikel zunächst die verschiedenen Organisationsmodelle, die pädagogischen Ziele sowie die historische Entwicklung von Ganztagsschulen in Deutschland. Anschließend stellt er den immer noch eher bescheidenen Stand der empirischen Forschung zur Ausbausituation, zur elterlichen Akzeptanz, zu den pädagogischen Gestaltungsformen und den Effekten von Ganztagsschulen vor. Abschließend werden ausgewählte Befunde aus einer vom Autor durchgeführten aktuellen bundesweiten Befragung von Schulleitungen von Ganztagsschulen aller Schulformen skizziert, die sich mit der Organisation, der Lernkultur sowie der Personalentwicklung an Ganztagsschulen beschäftigt hat.

In dem Beitrag von Falk RADISCH, Eckhard KLIEME und Winfried Bos werden die Ergebnisse einer empirischen Studie vorgestellt, die erstmals repräsentativ für Deutschland die pädagogischen Angebote und Schülerleistungen an Grundschulen mit und ohne Ganztagsangebot miteinander vergleicht. Dabei stützen sich die Autoren auf eine Sekundäranalyse der Daten der internationalen Grundschul-Leseuntersuchung IGLU sowie auf eine nachträgliche Schulleiterbefragung. Die Ergebnisse der Untersuchung machen deutlich, dass Ganztagsschulen zwar über ein breiteres Spektrum an Angeboten und Förder- 
maßnahmen verfügen, sich jedoch bei ihren Effekten hinsichtlich der Lesekompetenz der Schüler nicht von den Grundschulen ohne ganztägige Angebote unterscheiden.

In dem Beitrag von Karin BEHER und Thomas RAUSCHENBACH werden die konzeptionellen Elemente und Besonderheiten der offenen Ganztagsgrundschule in NordrheinWestfalen sowie erste Ergebnisse einer begleitenden explorativen Studie vorgestellt. Im Vergleich zu anderen Bundesländern zeichnet sich der Ausbau in Nordrhein-Westfalen dadurch aus, dass die Kooperation von Schule und Kinder- und Jugendhilfe unter systematischem Einbezug von Bildungs-, Betreuungs- und Erziehungselementen von Beginn an geregelt wird. Diese Entwicklungen werden aus dem Blickwinkel der Kinder- und Jugendhilfe betrachtet.

Thomas COELEN gibt in seinem Beitrag einen ersten Überblick über die Struktur und Organisation ganztägiger Bildungseinrichtungen in Finnland, den Niederlanden, Frankreich und Italien. Während die zentralen Strukturmerkmale dieser unterschiedlichen Bildungssysteme inzwischen relativ gut untersucht und beschrieben worden sind, fehlen jedoch noch komparative Studien, die die sozialpädagogischen Bildungs-, Freizeit- und Förderangebote in den in diesem Beitrag betrachteten ganztägigen Bildungssystemen in den Blick nehmen.

Abgerundet wird der Thementeil mit einer Sammelrezension von Grit ARNHOLD, in der wichtige Neuerscheinungen zur Ganztagsschulthematik vorgestellt werden. 